\title{
POLIITICA MONETARIA Y EQUIDAD EN COLOMBIA 1980-2010
}

\author{
Efraín Cuadro-Guzmán \\ Amaury Jiménez-Martínez
}


Panorama Económico, 24 (Octubre 2016 - Septiembre 2017), pp. 143-162

Efraín Cuadro-Guzmán

Amaury Jiménez-Martínez

\title{
Política monetaria y equidad en Colombia 1980-2010
}

\section{Resumen}

El presente artículo tiene por objetivo, analizar en qué medida la política monetaria, enfocada principalmente a controlar el comportamiento de la inflación, en las últimas tres décadas 1980 - 2010 , ha contribuido a mejorar los niveles de equidad en Colombia. Para lograr lo anterior, se hace uso de la observación de la trayectoria de las variables implicadas en el problema investigativo, detallando su tendencia en cada uno de los periodos estudiados, y haciendo la contrastación con el comportamiento de las demás variables endógenas al modelo. Uno de los hallazgos fundamentales de en este documento, se relaciona con el éxito de la política monetaria en Colombia durante el periodo en estudio, de acuerdo con las metas y objetivos planteadas por la autoridad monetaria. Sin embargo, otro de los resultados da cuenta del fracaso de la política económica en materia de equidad.

Palabras Clave: Política monetaria, equidad, ingresos, mecanismos de transmisión.

Clasificación JEL: E01, E21, E31, E52, E58.

\section{Monetary policy and equity in Colombia}

\begin{abstract}
This article aims to analyze the extent to which monetary policy focused mainly on controlling the behavior of inflation in the last three decades 1980 - 2010, has helped to improve the levels of equity in Colombia. To achieve this, use of the observation of the path of the variables involved in the research problem becomes, detailing its tendency in each of the periods studied, and by the contrast with the behavior of other endogenous variables to the model. One of the key findings in this document relates to the success of monetary policy in Colombia during the study period, according to the goals and objectives raised by the monetary authority. However, another result accounts for the failure of economic policy on equity.

Keywords: monetary Policy, equity, income, transmission mechanisms.
\end{abstract}

JEL Classification: E01, E21, E31, E52, E58.

\section{Politique monétaire et équité en Colombie 1980-2010}

\section{Résumé}

"Cet article analyse la politique monétaire pour contrôler l'inflation des dernières décennies 1980 à 2010 qui ont eu un effet bénéfique sur l'équité. L'observation du comportement à long terme de variables, avec le détail des tendances pour chaque période d'étude ainsi que le contraste avec d'autres variables du modèle. Une découverte est le bienfait de la politique monétaire durant cette période selon les buts et objectifs visés. Néanmoins un autre résultat démontre un échec en ce qui concerne l'équité.

Mots-clés: Politique monétaire, revenu, équité, mécanismes de transmission.

Nomenclature JEL: E01, E21, E31, E52, E58. 


\section{POLÍTICA MONETARIA Y EQUIDAD EN COLOMBIA $1980-2010$}

INFORMACIÓN DEL ARTÍCULO

Recepción de artículo: 25 de Junio de 2016

Concepto de evaluación: 1 de Agosto de 2016

Aceptación de artículo: 18 de agosto de 2016
Efraín Cuadro-Guzmán

Universidad de Zulia

Venezuela

Amaury Jiménez-Martínez

Universidad de Zulia

Venezuela

\section{INTRODUCCIÓN}

La principal preocupación de la economía debe ser el bienestar de la población. Es por esta razón que sus problemas fundamentales lo constituyen ¿Para quién, que y como producir bienes y servicios, con los recursos existentes en la economía, para la satisfacción de las necesidades? Si un sistema económico no puede resolver estos interrogantes, sin duda es un "sistema económico fallido".

Estas soluciones sistémicas, no son fáciles de generar, ante todo cuando se reconoce que los recursos económicos son relativamente escasos, de tal forma que satisfacer las necesidades de todos es un reto complejo que todo sistema debe afrontar. Los sistemas económicos de mercado tratarán de dar soluciones dejando que cada agente resuelva sus necesidades, hasta un grado de satisfacción medido según cómo le vaya en el mercado. En los sistemas económicos de corte socialista puro, es el Estado el que asume la responsabilidad de dar solución a las inquietudes fundamentales planteadas, y dará solución según la gestión, la tenencia de recursos y el sistema de distribución que se implemente en dicho régimen.

Esto último es determinante. Independiente del sistema económico que impere, si no está implementado un buen sistema de distribución o redistribución de la riqueza, inevitablemente el sistema se torna inequitativo y lo peor es que crea escenarios que profundizan y amplían cada vez más las brechas entre ricos y pobres y por tanto la inequidad se perpetua en dichos sistemas, en dichas estructuras, siendo mucho más difícil la consecución de las metas de propiciar una distribución de riquezas tal que la gran mayoría satisfaga sus necesidades básicas.

${ }^{1}$ Autor para correspondencia. Correo electrónico: efraincuadro@gmail.com; amauryjimenezmartinez@yahoo.com 
Desde la política económica se postulan como grandes objetivos el crecimiento, el desarrollo y la equidad, pasando por la estabilidad en los precios. A propósito de esto, se ha considerado que una economía estable en sus precios; es decir, tipos de cambio, tasas de interés, salarios y principalmente precios al consumidor, es una economía que invita a la inversión, que garantiza confianza y mejora la distribución de la riqueza. Colombia es uno de esos países que se ha acogido literalmente a este principio y lo cierto es que, es un país entre los Latinoamericanos, que se distingue en el largo plazo, por ser una de las macroeconomías mas estables.

Lo paradójico de lo anterior, es que a pesar de esta estabilidad de precios en general, Colombia ostenta una de las tasas de inequidad más alta, no solo de Latinoamérica, sino del mundo, paradoja que constituye el motivo de esta investigación, en donde se mostrará que mientras la estabilidad a la baja de los precios era una realidad para este país, tomando como referencia las últimas tres décadas, en este mismo periodo, la inequidad prácticamente se ha mantenido incólume, incluso en algunos periodos ha aumentado, muy a pesar de la tendencia a la baja de la inflación.

Este documento presenta una primera parte donde se perfila la investigación realizada; enseguida se describe la variable inflación en donde se mostrará la positiva tendencia de los precios a la baja; en la tercera parte de describen algunas variables que indican con contundencia la situación de inequidad del país: ingresos del $20 \%$ de mas altos ingresos, frente a los ingresos del $20 \%$ de los de más bajos ingresos de la economía. Se muestra también el coeficiente de Gini y la pobreza de las personas que están por debajo de la línea de U\$2 dólares diarios para poder vivir..... con lo que se espera contribuido en algo al debate sobre los efectos de la política monetaria sobre la equidad.

\section{METODOLOGÍA}

Uno de los principales postulados de los alcances de la política monetaria, es la de contribuir conjuntamente con la política fiscal y comercial, desde el ámbito macroeconómico, a conseguir grandes objetivos de política económica, tales como: pleno empleo de los recursos, estabilidad de precios, estabilidad cambiaria, crecimiento económico, desarrollo económico y por consiguiente, una mejor distribución de la riqueza generada, es decir, mayor equidad.

Lo ideal sería, conseguir paralelamente todos estos objetivos de política económica, pero la realidad no plantea este logro tan fácil. Desde hace mucho tiempo, Phillips planteó, por ejemplo, la disyuntiva entre empleo e inflación. Según este autor; si baja el desempleo, habrá más ingresos, lo cual generará más demanda de bienes y servicios, presionando los precios al alza. Mientras que si baja el empleo disminuirán los ingresos y con ello la demanda de bienes y servicios, presionando a la baja la inflación. La disyuntiva estaría en que, a mayor desempleo menor inflación y viceversa. Phillips supone que debería escogerse en el corto plazo, entre inflación con empleo vs bajos precios con desempleo.

Si la inflación tuviese sólo unas secuelas negativas en la reducción del poder adquisitivo de la moneda, lo cual podría ser cosméticamente "corregido" con ajustes en los salarios de los trabajadores, lo cual de por sí ya resulta un error de política económica, si sólo fuese así, se estaría en un escenario relativamente sencillo de manejar. El problema de fondo es que, 
además de no poderse corregir como se plantea arriba, la inflación casi siempreestá acompañada de un proceso de socavación de las intenciones del estado de propiciar una mejor o más equitativa distribución de la riqueza, condenando a los más pobres a la indigencia y perpetuando a los ricos en las cumbres de la mayor acumulación de riqueza, lo que significaría, que la inflación amplía la brecha entre pobres y ricos dentro de una determinada economía.

Colombia ha planteado claramente esta disyuntiva, optando por atacar de frente la inflación para así propiciar una mejor distribución de la riqueza, es decir, disminuir los niveles de inequidad existentes desde tiempos coloniales. La pregunta que se plantea esta investigación es ¿la política monetaria de las tres últimas décadas 1980 - 2010, dirigida expresamente a controlar la inflación, ha logrado o contribuido a mejorar los niveles de equidad en la economía colombiana?.

Bajo este contexto, en esta investigación se pretendióanalizar en quémedida la política monetaria, enfocada principalmente a controlar el comportamiento de la inflación, en las últimas tres décadas (1980 - 2010), ha contribuido a mejorar los niveles de equidad en Colombia. Para lo anterior, se procederá en primera instancia a describir cuál ha sido el comportamiento de la inflación en Colombia, cuál ha sido el comportamiento del nivel de equidad en Colombia en las últimas tres décadas 1980 - 2010 y en qué medida el control de la inflación como expresión de la política monetaria ha repercutido en el mejoramiento de la equidad.

En este sentido, de acuerdo a la taxonomía de Hernández Sampieri ${ }^{1}$, la investigación

\footnotetext{
1 Hernández Sampieri, Roberto. Fernández Collado, Carlos. Baptista Lucio, Pilar. Metodología de la Investigación. Colombia 1996. McGraw Hill.
}

que se desarrolló fue de tipo descriptivo, ya que se observa en ella la trayectoria de las variables implicadas en el problema investigativo, detallando su tendencia en cada uno de los periodos estudiados, y haciendo la contrastación con el comportamiento de las demás variables endógenas al modelo. Dada las dificultades para conseguir una serie de datos, especialmente, la referida a la equidad, la investigación sólo se enfoca en los últimos treinta años, hasta 2010.

\section{REFERENTES TEÓRICOS}

La política monetaria fue formalizada por economistas como Knut Wicksell, Irving Fisher y Clark Warburton (este último siendo conocido como el primer monetarista de la segunda postguerra) y Milton Friedman, entre otros, quienes le reconocieron un rol principal a la política monetaria, en la estabilidad macroeconómica. Los fundamentos de estos se hallan en ideas de economistas clásicos como John Stuart Mill, quien introdujo su principio que sugiere que el nivel general de precios se relaciona con la cantidad de dinero multiplicada por su velocidad de circulación. En contraposición al monetarismo, aún se halla el keynesianismo en cabeza de John Maynard Keynes y su énfasis en la importancia de la política fiscal en la estabilidad macroeconómica.

El debate a pesar de los años, sigue abierto entre monetaristas y keynesianos, pero hay consenso en que en el caso de la gran crisis de 1929, la política monetaria falló. Los clásicos creían que al reducirse la cantidad de dinero en la economía, también bajarían los precios y los salarios, y se recuperaría automáticamente al pleno empleo. No contaban con que precios y salarios fuesen rígidos a la baja. 
Gráfico 1. Esquema General de Mecanismos Generales.

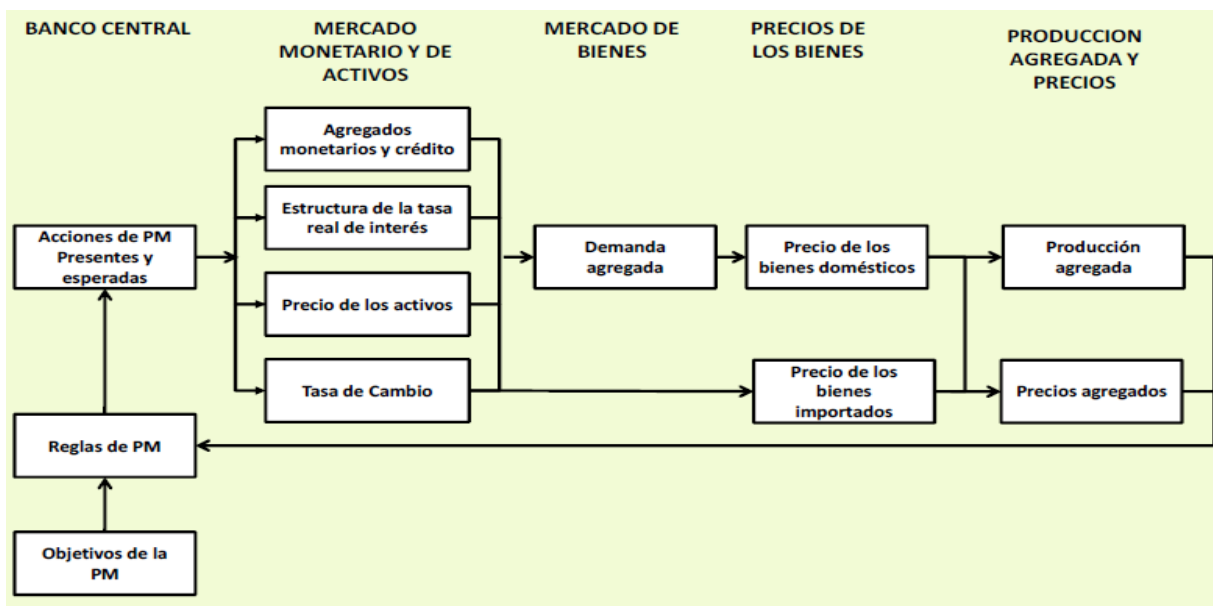

Fuente: Seminario de Política Económica y Estabilización. Docente Gerardo Castellanos. Unizulia 2013.

Si bien el intervencionismo fiscal entre los economistas es más pequeña ahora que a mediados del siglo $\mathrm{XX}$, recientemente en la crisis económica de 2008 -2009, la mayoría de gobiernos para salir de la crisis, aplicaron esencialmente medidas de estímulo económico, más en la línea keynesiana que en la línea monetarista.

Que tan "epidémica" puedan llegar a ser las decisiones de Política Monetaria y porqué canales se propagan dichas decisiones, ha sido el motivo de estudios y propuestas que hoy se aplican por parte de las autoridades monetarias de cada país. La forma como se estima que llegue la política a conseguir sus metas y objetivos, es lo que se denomina Mecanismos de Transmisión, y las diversas paradas intermedias que ella hace en el tramado macroeconómico hasta llegar a su cometido final, es lo que se conoce como canales de transmisión de la política (ver gráfico 1).

Keynes, por ejemplo, propuso el canal tradicional de la tasa de interés (ver gráfico 2). En su propuesta, partiendo del supuesto de perfecta sustitución entre bienes de capital y activos financieros, y suponiendo que los activos monetarios y no monetarios son sustitutos perfectos, la política monetaria se transmite a la demanda agregada, según Keynes, mediante una tasa de interés única, y la elasticidad de la demanda de dinero, es el único determinante de la efectividad de la política monetaria. De este supuesto se colige que una reducción de la cantidad de dinero aumenta el costo del crédito, reduciendo la inversión al eliminar proyectos marginalmente menos rentables e induciendo a una caída de la demanda. ${ }^{2}$

Por su parte Brainard y Tobin (1963) proponen el Canal de Activos (ver gráfico 3), como el ideal para que una medida de política monetaria, tenga éxito. Esta propuesta parte del supuesto que los activos financieros $y$ de capital son sustitutos imperfectos. "Esto implica que cambios inesperados en la oferta monetaria alteran los precios relativos,

2 Castellanos, Gerardo. Diapositivas IV Unidad. Curso de Política Económica y Estabilidad. Doctorado Ciencias Económicas. Universidad de Zulia. 
Gráfico 2. Mecanismos de transmisión por Tasas de Interés y por Créditos
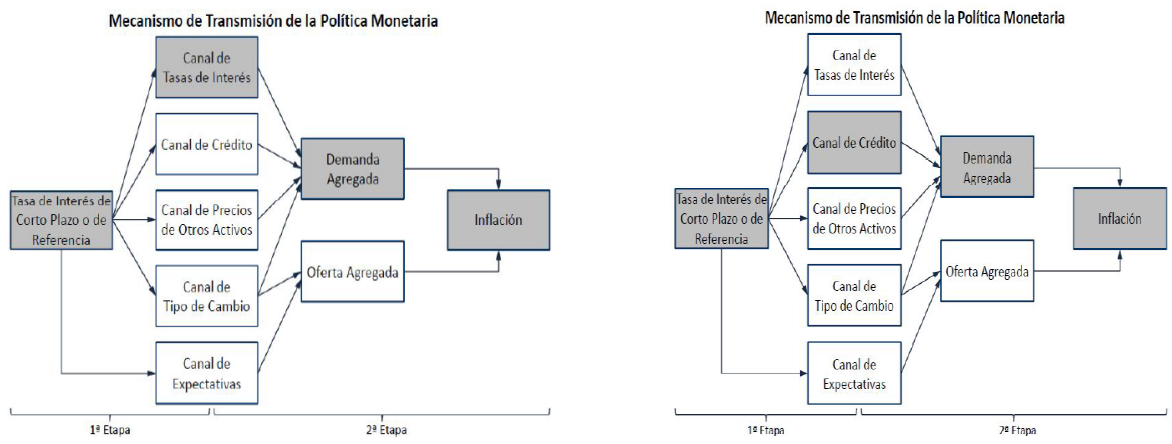

Fuente: Seminario de Política Económica y Estabilización. Docente Gerardo Castellanos. Unizulia 2013.

induciendo cambios en la composición del portafolio de los agentes y por consiguiente en sus decisiones de consumo e inversión. Por tanto, el impacto de la política monetaria puede ser entendido a través de la caracterización de cómo cambia la composición de la tenencia de activos. Finalmente llega a la conclusión que aumento en la tasa de interés disminuye el precio de los activos financieros, lo que puede reducir la riqueza y por lo tanto el gasto de los hogares y las empresas."

Teóricamente el Banco de la República valida el siguiente mecanismo de transmisión (ver gráfico 4): Cuando la inflación proyectada se ubica por debajo del rango meta, el Banco reduce sus tasas de intervención, lo cual induce a que caigan las tasas de interés del mercado, aumenta tanto la demanda agregada de bienes y servicios, como el $\mathrm{PIBr}$, paralelamente aumentan expectativas de inflación, efectivamente aumentan los precios y los costos, aumentando así la inflación. En el ámbito cambiario, al bajar las tasas de interés del mercado, se presenta salida de capitales y esto presiona a subir las tasas de cambio (devaluación), todo esto puede ocurrir con un rezago entre 12 y 24 meses.
Por el contrario, si la inflación proyectada se ubica por encima del rango meta, El Banco aumenta sus tasas de intervención, para provocar que suban las tasas de interés del mercado, disminuya tanto la demanda de bienes y servicios como el PIBr, bajando así las expectativas de inflación, y logrando efectivamente que bajen los costos y los precios y con ello la inflación. En lo cambiario, se esperaría una caída de los tipos de cambio (revaluación), todas estas consecuencias del mecanismo podría estar ocurriendo con un rezago de 12 a 24 meses $^{4}$.

El Banco de la república de Colombia, parte del convencimiento que

"una inflación baja y estable mejora el bienestar de la población. Esto tiene lugar de varias maneras: Una inflación baja promueve el uso eficiente de los recursos productivos. Por el contrario, cuando la inflación es alta una parte del tiempo de los individuos y una parte de los recursos de la economía se invierten en la búsqueda de mecanismos para defenderse de la inflación. Así por ejemplo, cuando la inflación es alta las empresas deben destinar más recursos al manejo de su portafolio para evitar pérdidas financieras. Estos son usos improductivos que no generan riqueza a la sociedad. 
Gráfico 3. Mecanismos de transmisión por Activos y Tipos de Cambio
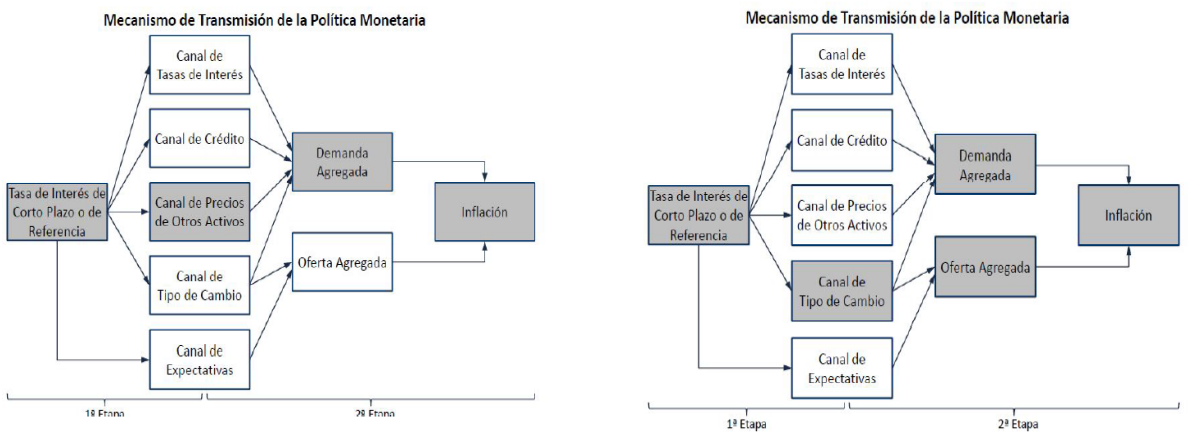

Fuente: Seminario de Política Económica y Estabilización. Docente Gerardo Castellanos. Unizulia 2013.

"Una inflación baja disminuye la incertidumbre. Se ha observado que las economías con alta inflación también padecen de una inflación más variable. La incertidumbre puede afectar negativamente la rentabilidad esperada de la inversión y por lo tanto el crecimiento en el largo plazo. La mayor incertidumbre implica también incertidumbre en los precios relativos, de tal manera que los precios pierden su contenido informativo sobre los precios futuros y los márgenes de comercialización aumentan. Todo esto afecta la asignación eficiente de los recursos y disminuye el crecimiento económico.

"Una baja inflación incentiva la inversión. Las decisiones económicas más importantes que toman los individuos y las empresas son, usualmente, decisiones de largo plazo: las decisiones de hacer una fábrica, de constituir una empresa, la decisión de educarse, de comprar vivienda. Estas decisiones dependen crucialmente del grado de incertidumbre sobre el futuro. Una inflación baja y estable es un indicador de estabilidad macroeconómica que contribuye a que las personas y las empresas tomen decisiones de inversión con confianza.

"Una inflación baja evita redistribuciones arbitrarias del ingreso y la riqueza, especialmente contra la población más pobre. Los asalariados y las personas jubiladas tienen menos mecanismos para protegerse de la erosión inflacionaria de sus ingresos. Por esta razón, una inflación creciente significa una redistribución del ingreso en contra de la población más pobre"s.

\section{Marco Institucional de la Política Monetaria en Colombia}

"Por mandato constitucional y legal el Banco de la República recibe la responsabilidad esencial de velar por el mantenimiento de la capacidad adquisitiva de la moneda. Es decir, el control de la inflación es objetivo principal de la política monetaria. De esta forma se reconoce que la estabilidad de precios es necesaria para el progreso económico y por lo tanto, el Banco debe ajustar el crecimiento global del dinero y del crédito con miras a lograr este objetivo. Aún más, la Ley 31 ordena a la Junta Directiva del Banco que al comenzar cada año fije metas de inflación que deben ser siempre inferiores a los resultados de la inflación del año precedente.

La política monetaria debe evaluar el estado de la economía en cada momento del tiempo y su posible evolución en los siguientes cuatro a ocho trimestres. Este es el horizonte de tiempo en el cual los cambios en las tasas de interés de intervención se transmiten a las otras tasas de interés e impactan la economía. Por lo tanto, la política monetaria opera necesariamente 
Gráfico 4. Mecanismos de Transmisión del Banco de la República Colombia: Cuando la inflación proyectada se ubica por debajo del rango Meta y Viceversa
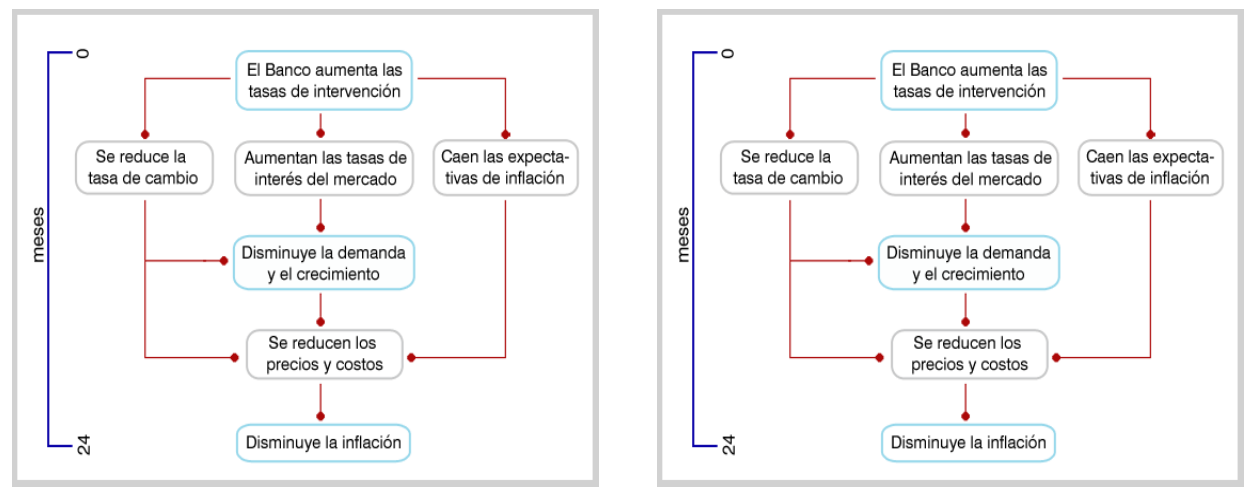

Fuente: www.banrep.gov.co

bajo un ambiente de incertidumbre. A ello contribuyen los siguientes factores:

La información sobre un buen número de variables económicas (por ejemplo, el PIB) está disponible con rezago y, en muchos casos, es sujeta a importantes revisiones y errores de medición. Otras variables importantes (como la brecha del producto o el crecimiento potencial de la economía) no son observadas y deben ser aproximadas a través de modelos e indicadores indirectos. En algunos casos es difícil distinguir el origen, los efectos, la persistencia y la magnitud de los choques que enfrenta la economía. Algunos ejemplos de choques son: Cambios en las tasas de interés externas o en los flujos de capitales. Incrementos de los precios internacionales del petróleo o del café. Reducción de la oferta de alimentos.

Además, el alcance y los efectos de la política monetaria no siempre son predecibles ya que dependen de: Las expectativas de los agentes sobre el comportamiento futuro de las tasas de interés, la tasa de cambio, la actividad económica y la inflación. La transmisión de las decisiones de política al resto de la economía ocurre con rezagos largos y variables, que pueden oscilar entre 12 y 24 meses. No siempre operan los mismos canales de transmisión, ni su potencia es constante. Adicionalmente, en una economía cambiante pueden surgir nuevos canales que antes no existían.
El objetivo primario de la política monetaria es alcanzar y mantener una tasa de inflación baja y estable, y lograr que el producto crezca alrededor de su tendencia de largo plazo. Esta es la única manera de lograr un crecimiento sostenido que genere empleo y mejore el nivel de vida de la población. Por el contrario, si la economía crece a un ritmo que no es sostenible, tarde o temprano se generará una crisis con consecuencias graves para la economía, deterioro de los indicadores sociales, pérdida de confianza de la población y caídas en la inversión y en el empleo"6.

\section{COMPORTAMIENTO DE LA INFLACIÓN COLOMBIA 1980 - 2010}

Se entiende por inflación el incremento generalizado, o en promedio ponderado, de todos los precios de los bienes y servicios de la economía, representados por una canasta básica de consumo, en este caso de consumo familiar.

La inflación puede provenir de un empujón de oferta o por uno de demanda, o por los dos simultáneamente. Cuando este impulso lo generan incrementos de los costos de producción en la economía (salarios, petróleo, combustibles, energía, 
o disminución de la producción), se dice que es inflación de oferta. Cuando el incremento de los precios de toda la economía tiene como causa inmediata la emisión de más dinero o una política monetaria expansiva o una política fiscal expansiva, entonces se identifica como inflación de demanda o de gasto agregado. Pero podrían confluir estas dos fuerzas de oferta y de demanda agregada, y conspirar contra la estabilidad de los precios propiciando ambas una inflación, entonces habrá que llamarla inflación por empujón de oferta y demanda agregada.

La realidad es que Colombia ha sido una de las economías en la región caracterizada por una relativa estabilidad de precios. A la sazón, es digno recordar, como en un discurso presidencial del entonces primer mandatario Dr. Virgilio Barco, cuando estaba siendo cuestionado por la inflación en Colombia que había subido en 1990 del 25\% al 29,3\%, él contestó que "la inflación en Colombia era alta, pero que aun así, la inflación de un año en Colombia, en ese momento era la de un día en varios países latinoamericanos, que registraban inflaciones anuales espectaculares: Argentina 2314\%, Brasil 2863\%, Perú 7481\% anual, entre otros. Por otra parte Dornbusch y Fischer en un afamado artículo en 1992, incluyó a Colombia entre un reducido número de países que se caracterizaban por lo que ellos denominaron "Inflación Moderada", es decir, inflación que ubicaron en un rango entre $20 \%$ y $30 \%$ anual.

Desde ya se puede comprometer este escrito al afirmar que siendo así, el problema de Colombia no ha sido ni de precios altos, ni mucho menos de inestabilidad de precios, sino de nivel de ingresos de los colom-

7 Dornbusch, Rudiger. Fischer, Stanley. Inflación Moderada. Ensayos Sobre Política Económica. Banco de la República. Departamento de Investigaciones Económicas. Junio 1992. № 21. bianos o mejor, del nivel de concentración del ingresos de los colombianos. En efecto en 1980, tal como lo muestra el gráfico 5, los precios crecían en Colombia un 25,6\%, manteniéndose una tendencia estable durante la década de los ochenta, tanto que el promedio de esta década fue del $23,5 \%$. Como se observa, la línea de tendencia fue una década en donde la inflación se pegó a ese promedio, se tornó inercial.

1991 inaugura una nueva institucionalidad para Colombia. La Asamblea Constituyente de ese año diseña una nueva Constitución, proponiendo un nuevo escenario normativo. En política monetaria, se replantea la organización del Banco de la República, desapareciendo la Junta Monetaria, quien regía la política monetaria y cambiaria, y nace la Junta Directiva del Banco de la República, que asume estas funciones, y se le da la responsabilidad con rango constitucional de controlar la inflación como objetivo número uno de la política monetaria. En esta década la inflación efectivamente empieza a bajar, el salario mínimo se comienza a negociar con las centrales obreras teniendo en cuenta la inflación esperada y la productividad laboral.

Además entre las normas constitucionales estaba la de comprometer al Banco a conseguir una "inflación meta" menor a la del año anterior. Con esta estrategia de política salarial y una fuerte política monetaria estrictamente ligada a permitir un crecimiento de la masa monetaria, acorde a la meta de inflación esperada, la nueva institucionalidad simbolizada por el nuevo rol del Banco de la República, estaba efectivamente consiguiendo, como dijo el ministro de hacienda de la época, Rudolf Hommes Rodríguez, "torcerle el pescuezo a la inflación", rompiéndole la inercia de la "inflación del 25\%" y poder así encaminar a Colombia a conseguir la meta de largo plazo de tener "inflación de un dígito". 
Gráfico 5. Comportamiento de la Inflación Colombia 1980 - 2010

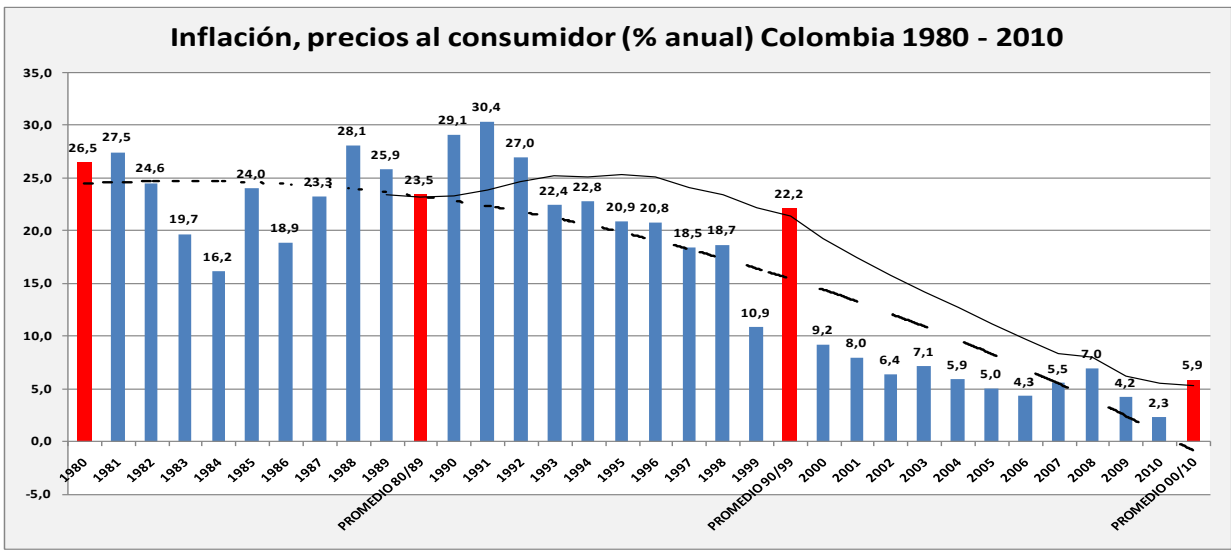

Fuente: Diseño autor. Datos Banco Mundial 2013

Vale resaltar que el promedio móvil a 10 años mostrado en la gráfica 1 presenta en los primeros años de la década del noventa, un incremento causado por los altos índices de inflación de finales de la década de los ochenta y de principio de los noventa, tendencia del promedio móvil a diez años que a mediado de este periodo se corrige para nunca más subir hasta lo que va de nuestros días. En efecto esta década cerró con un promedio móvil de $22,2 \%$, y una tendencia evidente a la baja, especialmente al final de la década, situación de caída de los precios que fue prohijada por la gran crisis de final de siglo, año 1999, en donde la demanda agregada bajó y la producción cayó a un $-4,2 \%$, caída histórica de la economía colombiana, que como era de esperarse, propició una mayor caída de los precios: del 18,7\% en 1998 al 10,9\% en 1999.

El nuevo milenio, el nuevo siglo en su primera década, la década de los "dosmiles", se caracterizó por una aceleración de los índices de inflación a la baja. Efectivamente, en el año 2000 los precios crecieron a 9,2\%, y al cierre de este periodo, esta variable solo llegó a 2,3\%. Resultado que puede destacarse como un éxito del diseño de la estrategia y de las políticas de control de la inflación en Colombia, ya que no sólo se han logrado estos índices deseados de "inflación de un dígito", sino que ésta ha bajado a índices impensados hace una década y lo que es más importante, se han logrado en medio de tasas de crecimiento económico, que si bien no son extraordinariamente altas, si han estado por encima del PIB tendencial. Es decir, que en medio de condiciones de bache inflacionario que podría haber elevado los precios, estos siguieron bajando significativamente, cerrando con un promedio móvil de los últimos diez años en solo $5,9 \%$ y con una tasa de inflación en el año 2010 digna de envidiar por cualquier país desarrollado de solo 2,3\%.

\section{COMPORTAMIENTO DE VARIABLES INDICADORAS DE EQUIDAD COLOMBIA 1980 - 2010}

El Banco de la república ha sido muy enfático en recalcarle a la opinión pública, que "Una inflación baja evita redistribuciones arbitrarias del ingreso y la riqueza, especialmente contra la población más pobre... Los asalariados y las personas 
jubiladas tienen menos mecanismos para protegerse de la erosión inflacionaria de sus ingresos...una inflación creciente significa una redistribución del ingreso en contra de la población más pobre"8. Es difícil contradecir estos postulados y alcances que plantea el Banco de la República, en consecuencia es de esperar que, dada una exitosa política monetaria en un periodo de treinta años, regulando la moneda y controlando la inflación, las condiciones de concentración de los ingresos, debía mejorar a favor de los más pobres. Observemos algunos indicadores.

Como se puede notar en la gráfica 6, en 1980 Colombia registraba una concentración del ingreso del 62,71\% en solo el $20 \%$ de las personas que obtenían los mayores ingresos de la economía. Es decir, que para el resto de las personas equivalentes al $80 \%$ de la población, solo les correspondía repartirse el 37,21\% de los ingresos. Esta era una terrible realidad de concentración e inequidad, que obligaba a las autoridades del estado en todos sus estamentos y a la empresa privada en todos los sectores económicos a comprometerse a mejorar estos indicadores.

Se pudiera atiborrar estas páginas de atenuantes y agravantes, pero loobjetivoes que después de treinta años, después de una nueva constitución (1991) mas social, más comprometida con los más necesitados, en el año 2010, el 20\% de las personas de mayores ingresos aún concentraba el $60,15 \%$, solo se había bajado dos puntos porcentuales, y por tanto el resto de la población (80\%), después de treinta años, solo accedía a repartirse $38,85 \%$ del total de los ingresos. Conclusión, los estratos de mas altos ingresos de la población, después de tres décadas de políticas públicas, y de una muy exitosa política monetaria,

8 Banco de la República prácticamente seguían concentrando proporcionalmente la misma cantidad de ingresos, un poco más del $60 \%$ del total.

La otra cara de la moneda, la más deprimente, y que confirma las tendencias perversas de la desigualdad o trampas de la pobreza en Colombia, se muestra en la gráfica No. 7, en donde se registra que $20 \%$ de las personas peor remunerados, solo llegaban en 1980, a acceder a un miserable $2,6 \%$ del total de los ingresos, es decir, que los ingresos del $20 \%$ de los peor remunerados, cabían proporcionalmente unas treinta veces en los ingresos obtenidos por el $20 \%$ de los mejores remunerados en la economía, o dicho de forma más contundente: que los ingresos de los mejor remunerados eran proporcionalmente 30 veces mayor que los ingresos de los más pobres.

Pero resulta más grave el hecho que después de treinta años de políticas económicas redistributivas, políticas fiscales y monetarias armonizadas supuestamente para reducir brechas sociales, después de todo este "esfuerzo", en el año 2010, esta población, el $20 \%$ de los peor remunerados, solo accedía todavía a un miserable 3\% del total de ingresos, y fue solo una pírrica mejora, porque realmente mantuvo en la última década estudiada, 2000 - 2010, un promedio solo el 2,36\% del total de los ingresos generados por la economía. En efecto la tendencia de largo plazo que se desprende de su comportamiento estadístico, es decreciente, lo cual quiere decir que en el periodo estudiado, cada vez esta población contrario a la justicia social esperada, presentó un menor nivel de participación de sus ingresos en la totalidad de los mismos en la economía colombiana (ver gráfico 7 línea punteada de tendencia).

La pobreza por ingresos tiene varios indicadores que la muestran. Estos datos que se observan en la Gráfica 8, denotan 
Gráfico 6. Ingreso del 20\% Mejor Remunerados en Colombia 1980 - 2010

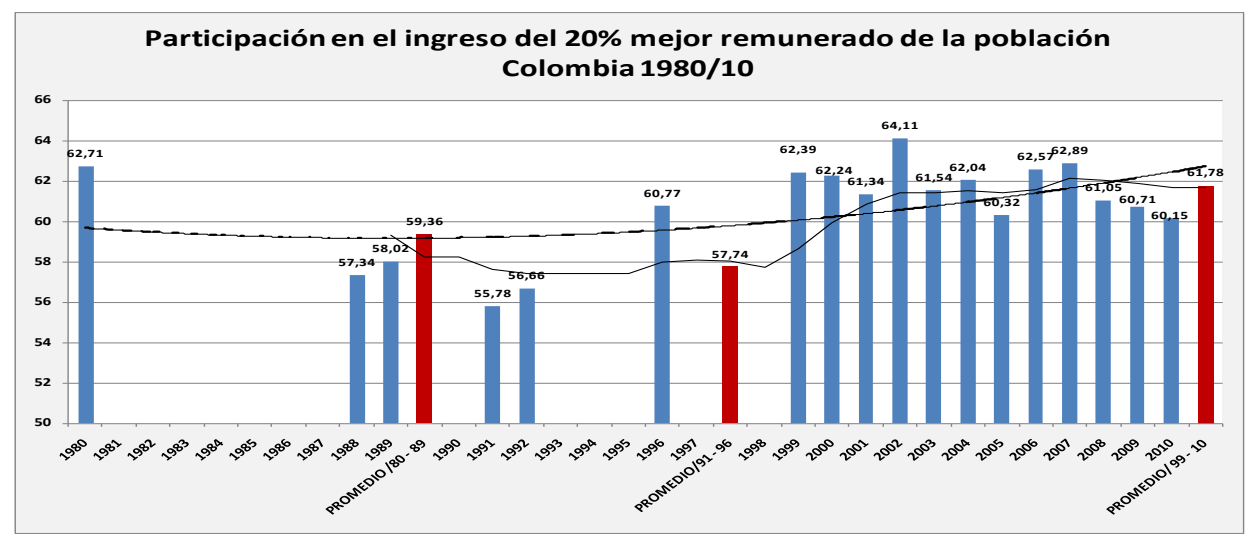

Fuente: Diseño autor. Datos Banco Mundial 2013

precisamente, que porcentaje de la población colombiana, devenga unos ingresos por debajo de $\mathrm{U} \$ 2$ diarios. A este nivel se le conoce como Línea de Pobreza, y consiste técnicamente en aquellas familias que sus ingresos son insuficientes para adquirir una canasta básica de bienes que contempla alimentación, vestuario y vivienda. En 1980 esa tenebrosa línea se trazaba en el $26,92 \%$, cerrando la década de los ochenta en 21,93.
El inicio de la nueva constitución y el proceso de apertura, coincidió con una baja de este índice, registrándose en esta decena anual un baja hasta el 17,82 en su promedio móvil. A raíz de la gran crisis hipotecaria y financiera en EE UU, y la caída de la producción, el empleo y los ingresos en todo el mundo en 1999, a lo cual no se pudo abstraer Colombia, efectivamente su $\mathrm{PIBr}$ cayó $-4,2 \%$, la pobreza se aumentó en los primeros años de la década de los noventas, pero

Gráfico 7. Ingreso del 20\% Peor Remunerados en Colombia 1980 - 2010

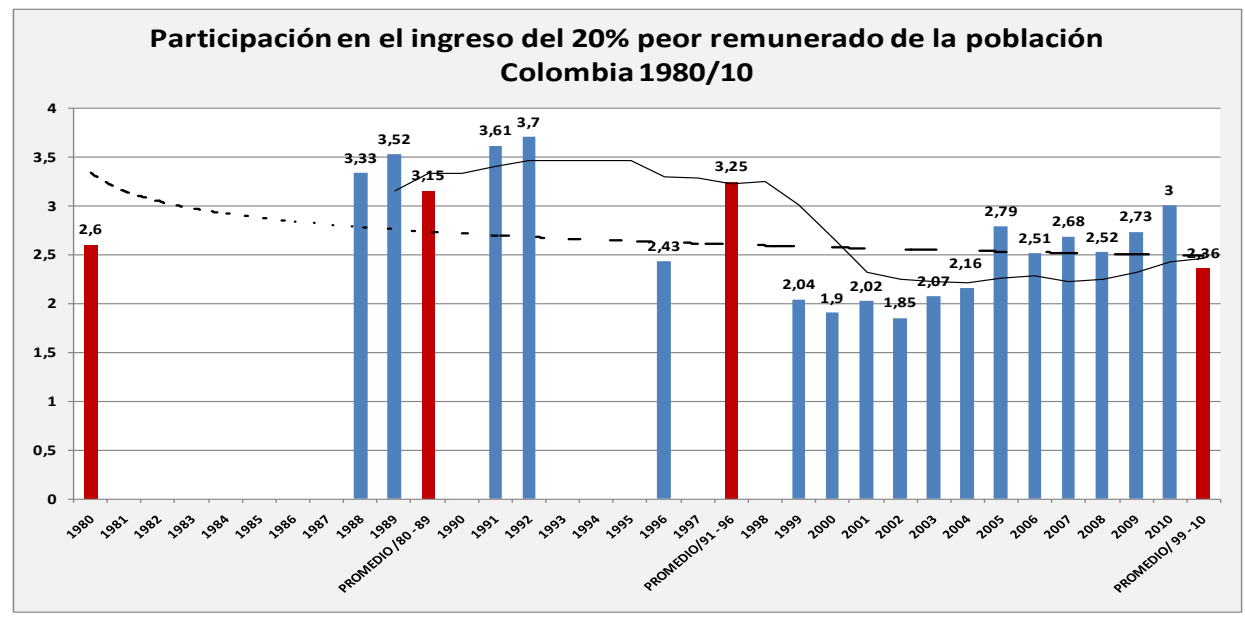

Fuente: Diseño autor. Datos Banco Mundial 2013 
Gráfico 8. Población Bajo la Línea de Pobreza en Colombia 1980 - 2010

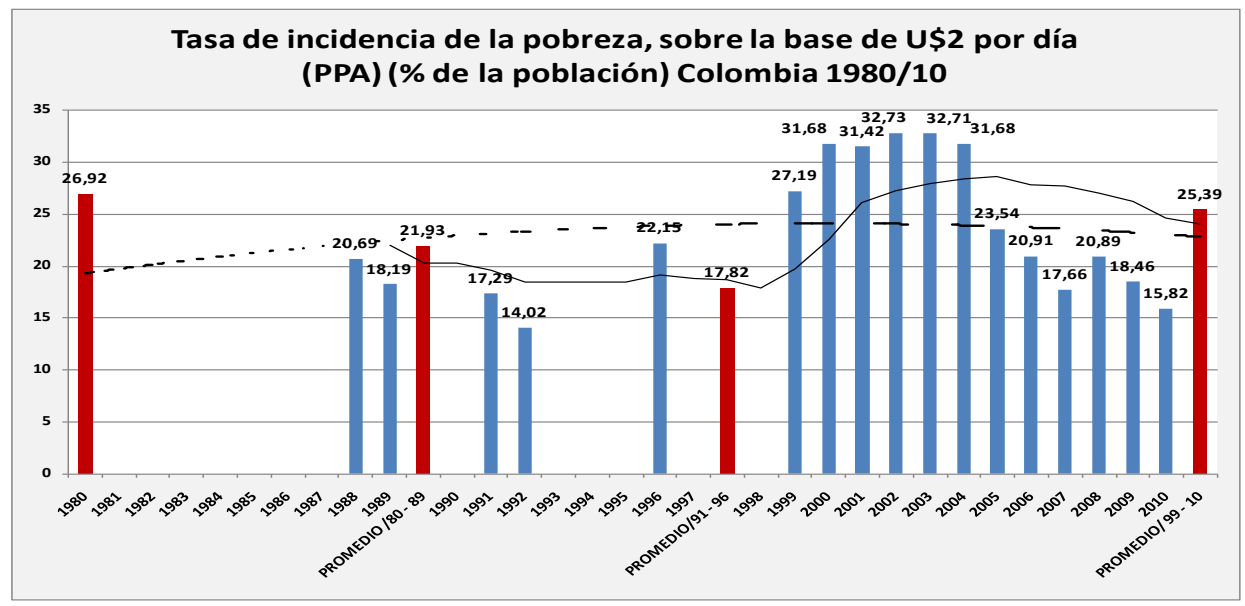

Fuente: Diseño autor. Datos Banco Mundial 2013

mejoraron los registros desde el año 2005 en adelante, una tendencia favorable a salir familias de la pobreza, bajando este registro al 15,82\% en el año 2010 , aunque por los altos registros acaecidos en el comienzo de la década, el promedio móvil a diez años se quedó remontado en un $25,39 \%$, pero con tendencia a la baja.

Bajando un poco más la cota de ingresos tal como se ve en el Gráfico 9, se halla la Línea de Indigencia, la cual denota aquellos hogares que perciben menos de $U \$ 1,25$ al día, es decir, aquellos que perciben ingresos insuficientes para adquirir siquiera una ración básica diaria de alimentos. Al respecto según datos del Banco Mundial, a 2010 Colombia tenía un 8,16\% de personal por debajo de esta línea, lo cual es alto, pero se debe reconocer que a partir del año 2005, este indicador mostró una espectacular caída, hasta estar en $8,84 \%$ en el año 2007. No obstante, el promedio móvil a diez años de la línea de indigencia, en la última década estudiada (1991 - 2010) nos deja un sabor amargo del $14,48 \%$, cifra estadísticamente explicable al ver por ejemplo, en el año 2002, un máximo del 20,26\% y el año 2010 bajar hasta un 8,16\%, tasas que se presentan en este mismo periodo.

Finalmente se presenta la medida clave de la inequidad: el Coeficiente de Gini ${ }^{9}$ Co-

9 El coeficiente de Gini mide con un solo número, el nivel de equidad o de inequidad, pues uno refleja el otro. En un plano cartesiano que relaciona en un eje el total de ingresos generados y en el otro, el total de población, se esperaría una recta diagonal de izquierda a derecha, que represente la perfecta distribución de la riqueza, donde por ejemplo, el $25 \%$ de la población accediera al $25 \%$ de los ingresos; que el $50 \%$ de la población accediera al 50\% de los ingresos y así sucesivamente. Pero no. "Esto no sucede ni en las mejores familias". Realmente predomina en las economías, independiente del sistema económico imperante y del grado de desarrollo del país, un determinado grado de inequidad en unos más que en otros. Es decir, donde el $25 \%$ de población, por ejemplo, acceda solo al $4 \%$ de los ingresos; que el $50 \%$ de la población de menores ingresos solo acceda al 10\% de los ingresos y en cambio, el $25 \%$ de población de mayores ingresos se quede con el $60 \%$ de los ingresos. Esta segunda situación predominante generaría en el mismo plano cartesiano descrito, una curva que se caracterizará por estar más separada de la recta de la perfecta distribución, en la medida en que haya más inequidad o más concentración de los ingresos en el grupo de mayores ingresos; o también se puede decir, que será más separada, en la medida en que haya menos ingresos entre las personas que obtienen los menores ingresos. Geométricamente se presentan entonces dos áreas: un área es el triángulo constituido en su hipotenusa, por la recta de la perfecta distribución de la riqueza, y el otro área es el que se formaría entre la recta de la perfecta distribución de la riqueza y la curva cóncava que surge de la inequidad, y que se conoce como la Curva de Lorenz. El Coeficiente de Gini es en consecuencia obtenido de relacionar 
Gráfico 9. Población Bajo la Línea de Indigencia Colombia 1980 - 2010

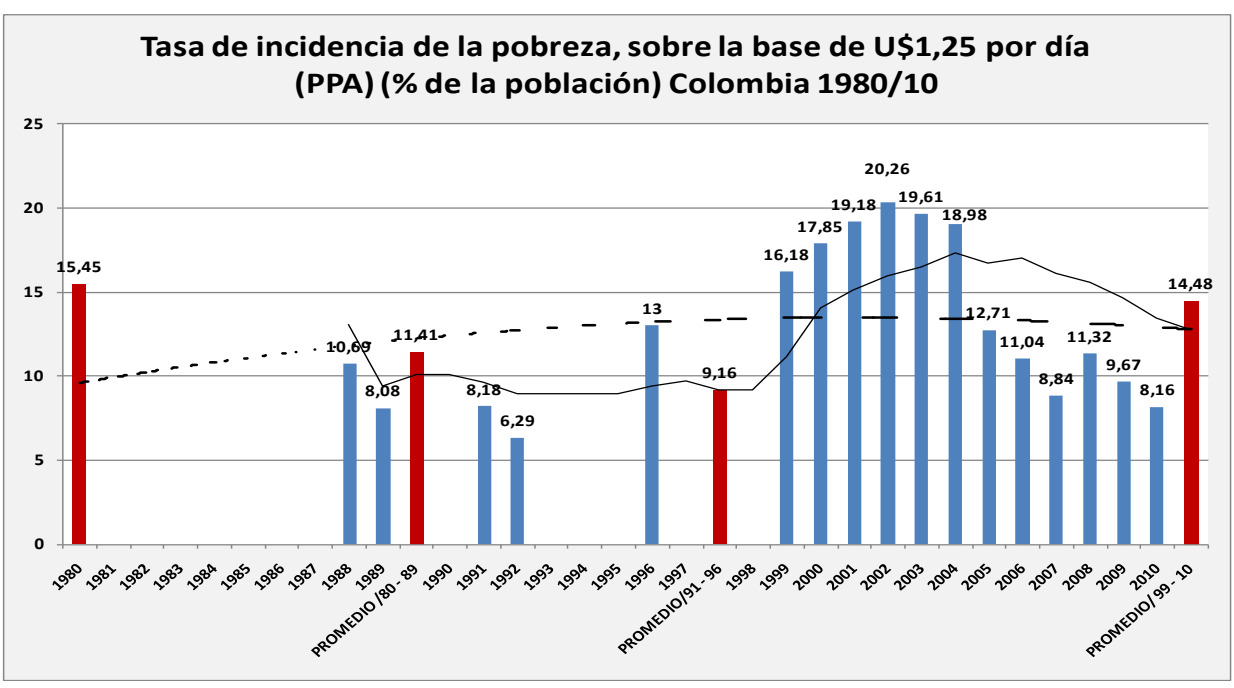

Fuente: Diseño autor. Datos Banco Mundial 2013

lombia ha presentado históricamente, un Gini relativamente alto, razón por la cual siempre se le cataloga como una de las economías más inequitativas. Esta realmente es una herencia perversa que las generaciones subsiguientes de líderes y autoridades colombianas en todos los campos, no han tenido la voluntad política de liquidar.

La historia de Colombia nos muestra una sociedad polarizada desde el mismo momento del proceso de colonización, situación que fue común a todos los países latinoamericanos. La posterior etapa de emancipación colonial solo dio origen a unas nuevas castas sociales en las naciones emergentes, que detentaron el poder económico, y con él, el poder político, relegando a los indígenas, negros, mestizos y blanco criollos empobrecidos, a condiciones de marginalidad social, es decir, sin acceso a educación, salud,

(dividir), el área de inequidad del plano cartesiano, sobre el área del triángulo de la perfecta distribución de los ingresos. Gini estaría entonces entre cero y uno y mientras mayor sea Gini, mayor será la inequidad, o mayor será el grado de concentración de la riqueza en dicha economía. vivienda digna, ni oportunidades. Todos los países de Latinoamérica registran este dramático cuadro de sucesos concatenados, que configuraron unas sociedades injustas e inequitativas que hasta nuestros días muestra sus negativas secuelas en unos países más que en otros.

Colombia en 1980 mostraba un Gini del 59,13 (ver gráfico 10), relativamente alto. Esto significaba en ese entonces, comienzo de la década de los ochenta, un nivel muy alto de concentración de la riqueza, fenómeno que está explicado, además de las condiciones estructurales históricas, del enquistamiento de unas clases sociales que detentan el poder económico, social y político, casi que por razones de herencia, y por tanto generan conscientes o inconscientes, procesos de exclusión socioeconómica y política, además de estas razones, se pueden encontrar otras. Entre estas están las brechas salariales, o por remuneración al trabajo, donde unos pocos devengan mucho, y muchos (la gran mayoría) devengan muy poco. También encontramos 
la brecha entre las remuneraciones al capital y a la tierra, versus la remuneración al trabajo: esta inequidad se halla cuando en términos generales se gana menos por ofrecer al proceso productivo su fuerza laboral (trabajo), frente a ofrecer al proceso de producción del país, su capital acumulado o sus tierras.

Otra causa de injusticias en la distribución delariqueza deunaeconomía, seencuentra en las grande diferencias de dividendos entre las grandes y medianas empresas que se conforman, generalmente beneficiadas por intervenir mercados monopólicos $\mathrm{u}$ oligopólicos, en donde ejercen todo su poder dominante determinando precios, asignando recursos a su antojo, este semejante "Goliat Económico", frente a un enjambre de microempresas que obtienen solo ingresos de subsistencia o en el mejor de los casos solo generan ingresos para un proceso raquítico de acumulación simple de capital, marcando con ello, una gran inequidad por tamaño de empresas. A lo anterior se le agrega un agravante, pues estas grandes o medianas empresas nacionales, generalmente nacen bien articuladas a corrientes del comercio internacional desde donde pueden acceder a mayores ingresos, dado un proceso favorable devaluacionista, todo esto frente a ese mismo enjambre de microempresas que solo cuentan con mercados barriales, locales... en fin, mercados muy limitados en su tamaño.

Repasar estas condiciones generales que subyacen a un proceso de deterioro de la equidad o para prohijar la mala distribución de la riqueza, pareciera estar repasando desafortunadamente los hechos que han marcado la historia económica de Colombia. En efecto, después del periodo de dominación colonialista español, Colombia entró en una etapa de reacomodamiento de una clase emergente criolla que concentró el poder. Posteriormente vino una etapa marcada por políticas de "Protección a la Industria Naciente" para supuestamente "lograr Sustituir las Importaciones", todo en medio de una posición de economía caracterizada por enclaves socioeconómicos de concentración de la riqueza, y en condiciones de economía satélite de una potencia mundial, satélite que gravitaba desde la primera postguerra y aun lo está, alrededor de una economía centro - dominante - potencia económica, como lo es los Estados Unidos de América.

De 1980 hasta el término de esta década en 1989, y extendiendo la mirada hasta 1991/92, es esperanzador ver que la línea de tendencia de esta treintena de datos de inequidad, disminuyen mostrando que la inequidad está cediendo, que el ingreso se comienza a distribuir mejor entre los colombianos (ver gráfico 10 línea punteada de tendencia de largo plazo). Pero de una vez llegado 1996, se esfuman esas esperanzas elevándose nuevamente el coeficiente de Gini, situación que se empeora hasta cerrar la década, el siglo y el milenio, con una inequidad (Gini de 50,84) casi igual a la registrada 20 años atrás en 1980. Esto se explica en parte por las altas tasas de desempleo, fruto de la peor crisis sufrida por la economía colombiana en los últimos 100 años (caída del 4,2\% del PIBr), cierre de muchas empresas y en general, la caída de los ingresos y del patrimonio de los colombianos. Como siempre sucede, los más vulnerables en estas crisis sufren más, quienes habían podido con mucho esfuerzo escalar a ingresos medios bajan a ingresos bajos y quienes habían salido de la indigencia y la pobreza vuelven a estas denigrantes condiciones, pagando con creses su "atrevido y fugaz" ascenso socioeconómico. Paralelamente, quienes detentan el poder económico se defienden mejor, y el resultado final es que hay más 
Gráfico 10. Inequidad Medida por Índice de Gini Colombia 1980 - 2010

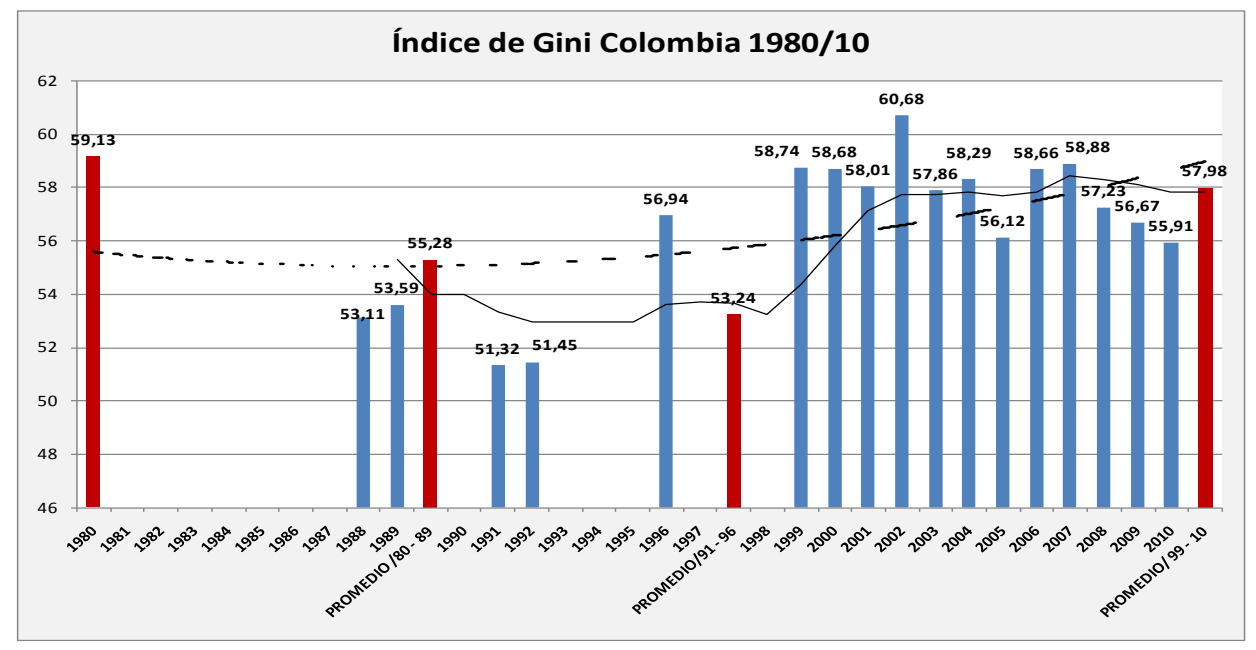

Fuente: Diseño autor. Datos Banco Mundial 2013

pobres que antes frente al mismo número de ricos, situación objetiva para aumentar los indicadores de inequidad, tal como lo muestra el coeficiente de Gini de Colombia.

En toda la primera década de los "dosmiles", último decenio en estudio, se observa un mantenimiento de las condiciones de inequidad, con solo un leve descenso hasta registrar un enano descenso del Gini en el cierre del año 2010 de 55,91, pero el trago amargo lo constituye el promedio móvil de la década que quedó trepado en un Gini de 57,98, casi 58, muy parecido al Gini de 1980, treinta años atrás, que llegó a ser 59,13... ¡tres décadas perdidas!

El agravante de estas desalentadoras cifras de inequidad, es que ellas tristemente se logran en un década que en general la economía colombiana mostró un crecimiento de la producción y de los ingresos, por encima de la tendencia de largo plazo, es decir, crecimiento por encima del $4 \%$, tasa de crecimiento que ha marcado la tendencia del PIBr en los treinta años estudiados. Pero además se presentaron estas negativas cifras de inequidad, en un periodo en donde la inflación cayó a cifras realmente espectaculares, empezando el decenio con una inflación del 9,2\% en año 2000 y terminando en el 2010 con un incremento de los precios de solo $2,3 \%$.

Unas reflexiones apenas de sentido común de los hechos demostrados a estas alturas del documento, es que ante un aparente éxito de la política monetaria, que se refleja de forma evidente en una inflación realmente controlada a partir de la nueva Constitución Nacional de 1991, con nuevas funciones asumidas por la Junta Directiva del Banco de la República, no se ha reflejado, al menos de forma evidente, tal como lo buscaba la política monetaria regentada por el Banco de la República, en unas mejores condiciones de distribución de la riqueza.

La última década estudiada es un excelente escenario para hacer la anterior afirmación. Pareciera que la política monetaria hubiese padecido una o varias de las siguientes afecciones: 
No haber sido armónica con las demás políticas fiscales, comerciales o de choques de oferta agregada, o por el contrario, que estas políticas no se armonizaron con los buenos resultados de la política monetaria, en función de que los efectivos controles a la inflación alcanzados se tradujeran en más equidad entre los colombiano. O pudo haber sucedido que la política monetaria haya conseguido sus metas de inflación solo a costa de sacrificar el salario de los más vulnerables de la economía, manteniendo los niveles de ingresos de los más acaudalados del país. O que el crecimiento económico registrado en Colombia en los últimos años, hubiese estado marcado por un uso intensivo de capital y poca mano de obra, al igual que hubiese estado concentrado en sectores económicos enclaves articulados con el sector externo, generando mucha remuneración al capital y muy poca remuneración al trabajo. O que las condiciones favorables de inflación y sus buenas consecuencias en términos de propiciar más inversión, se hubiesen conseguido, pero en la atracción de inversión nacional y extranjera, que contrario a lo esperado, se haya caracterizado dicha inversión, por ser intensiva en capital, solo mejorar los dividendos de las empresas, generar poco empleo, y que hayan trasvasado regalías hacia sus casas matrices en el exterior, más que buscar permear con estos ingresos a toda economía nacional, lo cual no propicia un proceso de incremento significativo del ingresos de los más pobres, donde se hubiese evitado, lógicamente, lo que ha sucedido: mantenimiento de los niveles de desigualdad en Colombia en los últimos 30 años estudiados.

Duele decir que desafortunadamente algo de todos los diversos síndromes antes propuestos, se han presentado en Colombia, en la última década con mayor fuerza. Se empieza por decir que a pesar de que ha habido un crecimiento del sector minero energético, construcción y financiero, por el lado de la oferta de productos agropecuarios, se ha adolecido de una política de choque de oferta que redunde en el mejoramiento de sectores rurales que realmente son los que más determinan las inequidades de la economía colombiana. Otro aspecto aparentemente cosmético se ha presentado en la negociación del salario mínimo en Colombia, al cambiar a partir de 1992, su soporte de negociación, en ese entonces se dejó de considerar la inflación del año anterior, para empezar a considerar la inflación proyectada para el siguiente año, como indicador de mayor fuerza para negociar el incremento del salario de los más vulnerables; el efecto ha sido un factor menos de inflación de costos, a costa de unas mejoras salariales realmente pequeñas en comparación con los avances de la economía como un todo; de igual manera en este campo, se han implementado reformas de flexibilización laboral que han bajado los costos para el empleador, incidiendo positivamente en quitarle presión a la inflación de costos, y que no han significado más empleos formales como se pretendía. De igual manera, se ha dado un crecimiento determinado en gran medida por el crecimiento de grandes empresas intensivas en capital y tecnología de punta, en desmedro del uso intensivo de la mano de obra, lo que está evidenciado en el crecimiento de la petroquímica y sectores conexos, los cuales efectivamente, tiene como nicho naturales de mercado, los mercados externos.

Colombia por otro lado, registra la tercera posición en materia de crecimiento de la inversión extranjera en Latinoamérica, atracción que se presenta especialmente por la estabilidad macroeconómica que exhibe, ya que por seguridad y orden público, 
es evidente que no es la mejor opción. Desafortunadamente esta inversión no ha llegado a significar aún, grandes saltos positivos de elevación de ingresos reales de los más pobres, y más bien se han venido conectando a enclaves económicos con las mismas características sobresaltadas en renglones anteriores atribuidas a las grandes empresas nacionales, favoreciendo en términos generales la concentración de la riqueza de la economía colombiana.

\section{CONCLUSIONES}

La política monetaria en Colombia ha sido exitosa, si se comparan los fundamentos teóricos aplicados, las metas y objetivos propuestos, frente a las metas y objetivos logrados. Efectivamente, desde 1991 donde la inflación en Colombia era de 30,4\%, y ha bajado en el 2010 a solo 2,3\%. Espectacular.

En materia de equidad, la política económica en general no ha sido exitosa. El 20\% de las personas de mayores ingresos siguen después de una treintena de años (1980 2010), concentrando un poco más del $60 \%$ de los ingresos, mientras que el $20 \%$ de las personas de menores ingresos, en este mismo periodo, aun sigue accediendo solo a un 3\% del total de los ingresos, marcándose una brecha muy amplia de ingresos y por tanto de inequidad.

Los niveles en que ha bajado tanto la línea de pobreza como la línea de indigencia, son "victorias pírricas". Siendo objetivos, y frente al supuesto esfuerzo realizado por el Estado Colombiano, se podría afirmar que las condiciones de pobreza e indigencia, después de treinta años, siguen prácticamente igual.

Los pésimos logros en materia de equidad se corroboran de manera expresa, cuando se muestra el comportamiento del coeficiente de Gini que en cierta forma resume los indicadores antes resaltados. Los datos nos muestran que el grado de desigualdad en 1980 era de 59,13 mientras el promedio de los diez últimos años de la serie estudiada, años "dosmiles", fue de 60. El año 2002, por ejemplo, el retroceso en materia de inequidad fue tal que el coeficiente de Gini fue 60,68. Aberrante, especialmente cuando en estos años se lograron los mayores índices de crecimiento económico por encima de la tendencia. Es decir, se generó riqueza pero se concentró aun más su tenencia...lamentable.

Lo anterior quiere decir que el alcance que el Banco de la República le atribuye a la consecución por parte de la política monetaria de una Inflación baja, simplemente no se honró. Es decir, la inflación baja en Colombia, no evitó las redistribuciones arbitrarias del ingreso y la riqueza, en desmedro de la población más pobre, manteniendo las mismas condiciones de inequidad de hace treinta años.

Quedan para la agenda investigativa tres dudas: ¿será que los principios con los que se elaboran las políticas monetarias en Colombia están desenfocados de la realidad socioeconómica del país? o ¿será que la política monetaria ha estado bien enfocada, que el Banco de la República ha realizado bien su tarea, pero que son las demás políticas, fiscales, comerciales y laborales, entre otras, las que no han coadyuvado a "torcerle el pescuezo" a la inequidad en Colombia tal como se lo torció a lainflación la constitución de1991? o ¿será que variables exógenas o metaeconómicas, tales como el conflicto armado persistente en Colombia, mas la corrupción político - administrativa, esto unido a una clase empresarial con una cultura arraigada de explotación del trabajador, moviéndose además en estructuras de mercado 
monopólicas de enclaves, son entre otras variables metaeconómicas, los factores que han incidido en que las condiciones de inequidad en Colombia se mantengan, muy a pesar del éxito de las políticas monetarias? ...Se deja la agenda abierta

\section{REFERENCIAS BIBLIOGRÁFICAS}

Arango Londoño, Gilberto. Estructura económica colombiana. McGraw Hill. 2000. Bogotá - Colombia.

Cárdenas S. Mauricio. Introducción a la Economía Colombiana. Alfaomega. 2013. Bogotá - Colombia.

Cuadrado Roura, Juan R. Política Económica. Objetivos e Instrumentos. McGraw Hill. 2000. Madrid - España.

Cuadrado Roura, Juan R. y otros. Introducción a la Política Económica. McGraw Hill. 1997. Madrid España.

Chatterjee, Satyajit. ¿Porqué tiene importancia la Política Monetaria Contracíclica? BOLETÍN. VOLUMEN XLVII, N 4. Octubre-Diciembre de 2001. CENTRO DE ESTUDIOS MONETARIOS LATINOAMERICANOS. CEMLA. México - México.

Dornbusch, Rudiger. Fischer, Stanley. Inflación Moderada. Ensayos de Política Económica. № 21 junio 1992. Pág. 7-67. Banco de la Re- pública. Departamento de investigaciones Económicas. Bogotá _ Colombia.

Hernández Sampieri, Roberto. Fernández Collado, Carlos. Baptista Lucio, Pilar. Metodología de la Investigación. McGraw Hill. 1996. Bogotá - Colombia.

Kalmanovitz, Salomón. Economía y Nación una breve Historia de Colombia. Cuarta edición corregida y aumentada. TM Editores. 1998. Bogotá - Colombia.

Kalmanovitz, Salomón. Nueva Historia Económica de Colombia. Taurus. 2010. Bogotá Colombia.

Méndez Ibisate, Fernando. SETENTA AÑOS DE LA TEORÍA GENERAL DE KEYNES. UNA REVISIÓN CRÍTICA

Ocampo Gaviria, José Antonio. Otros. Historia Económica de Colombia. Edición Revisada y actualizada. Planeta.2007. Bogotá - Colombia.

Sarmiento Palacio, Eduardo. Apertura y Crecimiento Económico. De la desilusión al Nuevo Estado. TM editores. 1996. Bogotá Colombia.

Zalher, Roberto. Política Monetaria y Financiera.

Cuadro-Guzmán, E., \& Jiménez-Martínez, A. (2016). Política monetaria y equidad en Colombia 1980-2010. Panorama Económico, 24, 143-162.

\section{AUTORES}

\section{Efraín Cuadro-Guzmán}

Doctorante en Ciencias Económicas de la Universidad de Zulia (Venezuela). Economista y Especialista en Gestión del Comercio Internacional y Desarrollo Industrial de la Universidad de Cartagena. Actualmente Docente/Investigador de la Facultad de Ciencias Económicas de la Universidad de Cartagena (Colombia) y miembro del grupo de investigación Mercado Laboral. Adicionalmente, se ha desempeñado como conferencista y consultor, así como también se desempeña como Vicerrector Administrativo de la misma Universidad. 